\title{
A Comparative Study of Lipid Profile among Smokers and Non Smokers
}

\author{
Nabin Kumar Byar, Tirtha Man Shrestha, Narayan Prasad Pratap, Santosh Khanal \\ ${ }^{1}$ Department of General Practice and Emergency Medicine, Maharajgunj Medical Campus, Tribhuwan \\ University, Nepal
}

\section{Corresponding Author:}

Dr. Nabin Kumar Byar

E-mail: byarnabin@gmail.com

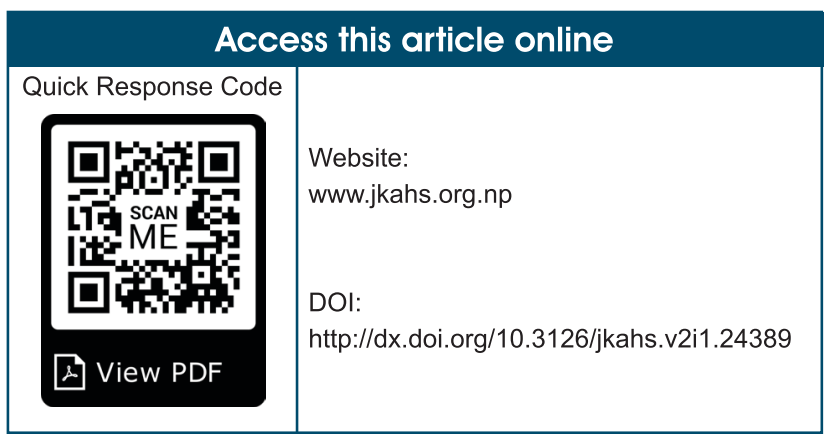

\section{ABSTRACT}

Introduction: Smoking is a risk factor for the development of cardiovascular diseases and many other diseases. Smoking is found to alter lipid profile and poses a risk for atherosclerosis. This study aims to compare lipid profile between smokers and non smokers.

Methods: Hospital based observational study was carried out in Tribhuwan University, Teaching hospital , Kathmandu, Nepal on 90 smokers and 90 non smokers who were apparently healthy. Data on socio demographic variables were collected using a questionnaire and lipid profile was estimated on fasting venous blood in an auto-analyzer. The lipid levels of smokers and non smokers were compared using t-test .

Results: Most of the smokers were daily wagers and they consumed alcohol and high fat food compared to non smokers. The serum levels of HDL were significantly lower in smokers compared to nonsmokers.LDL level was positively correlated with numbers of cigarettes/ beedi smoked. The LDL level was significantly high in those who smoked more than 10 cigarettes or beedis. The duration of smoking had no significant effect on lipid profile. LDL and VLDL were significantly high among those with pack years more than 3 . LDL levels positively correlated with pack year.

Conclusion: Tobacco smoking is associated with dyslipidemia (increased LDL-c and decrease HDL-c level.

Keywords: Dyslipidemia, HDL; LDL; Lipid profile; Smoking.

\section{INTRODUCTION}

Tobacco smoking is one of the most potent and prevalent addictive habits and is practiced by about a third of world's population. The National Demographic Health Survey conducted in 2006 reveals that nearly one third of males (32.5\%) in Nepal and while $15.2 \%$ of females smoke cigarettes.

Smoking is a major risk factor for Atherosclerosis and Coronary Heart diseases. ${ }^{1}$ Among the many 
risk factors associated with the development of atherosclerosis, the principle cause is dyslipidemia. ${ }^{2}$ Smoking harms nearly every organ of the body, causing many diseases and reducing the health of smokers in general. Smoking is a risk factor for development of cancer in every organ of the body, cardiovascular diseases, abdominal aortic aneurysm, respiratory illness, decreased fertility, congenital diseases, peptic ulcer diseases, periodontal diseases and many other diseases. ${ }^{5}$ Smoking is found to cause increase in the concentration of total serum cholesterol, triglycerides, LDL-cholesterol, VLDLcholesterol and fall in the levels of HDL cholesterol, as reported by various studies..$^{3-7} \mathrm{~A}$ study conducted by Afshan et al in Konaseema Institute of Medical Sciences, Amalapuram, india in 2011 among 50 smokers and 50 non smokers regarding comparison of their lipid profile showed that the serum TC, TG, LDL and VLDL were higher in smokers as compared to non smokers and the serum HDL level was significantly decreased in smokers compared to non smokers showing greater risk of these persons to atherosclerosis and coronary heart disease. ${ }^{9}$ This study aims to compare VLDL, LDL, TG, and HDL between smokers and non smokers and to study the difference in level of VLDL, LDL,TG and HDL on the basis of severity of smoking.

\section{METHODS}

A hospital based observational study was carried out in patients attending general health check up OPD at Tribhuwan University Teaching Hospital, Kathmandu. Patients were recruited from July 2016 to September 2017 after taking approval from Institutional Review Board of TUTH.

Inclusion Criteria: Age: 21 years-50 years

Non smokers: who never smoked

Smokers: Smokers are defined as those who had smoked at least once every week for last one year and were further classified as:

Mild smoker: 1-10 cigarettes/day Moderate smoker: 10-15 cigarettes/day Severe smoker:15-20 cigarettes/ day
Exclusion Criteria: Diabetes and Endocrine disorders, Hypertension, Renal disorders, Coronary Artery diseases, History of drugs intake: $\beta$ Blockers, lipid lowering drugs, steroids, history of alcohol Intake and drugs abuse.

Non-Probabilistic sampling method was used for sampling andsample size was calculated using the formula

$$
\mathrm{n}=\frac{2\left(\mathrm{z}_{\alpha}+\mathrm{z}_{\beta}\right)^{2} \mathrm{~s}^{2}}{\mathrm{~d}^{2}}
$$

where

$$
\begin{aligned}
& z_{\alpha}=1.96 \text { at } 96 \% \text { confidence level } \\
& z_{\beta}=1.28 \text { at } 90 \% \text { power }
\end{aligned}
$$

$\mathrm{S} 2=$ pooled variance calculated using $\mathrm{S} 1$ and $\mathrm{S} 2$. $\mathrm{S} 1=6.9$ and $\mathrm{S} 2=9.87, \mathrm{~d}=$ difference to be detected (assume $d$ to be 4 unit difference in mean HDL). By using this formula the sample size is $n=90$ in each group, with a total sample size of 180 .

Subjects were explained in detail about the study and written informed consent was collected. Detailed history of smoking in terms of number of cigarette smoked per day, and duration of smoking and other relevant history like any co-morbidities or any drug intake was taken using an investigator administrated semi structured questionnaires. Blood for lipid profile estimation which includes: serumcholesterol, serum triglyceride, High density Lipoprotein, Low density Lipoprotein, and Very low density lipoprotein was drawn by nurses after the overnight fasting under all aseptic precautions. Blood samples were sent to IOM TUTH biochemistry laboratory and analyzed.

All data were entered in SPSS v.22 for analysis. Frequency and percentage were calculated for categorical variables. $\mathrm{P}$-value $<0.05$ was considered as statistically significant.

\section{RESULTS}

Out of 180 samples, most of the respondents (41.1\%) were of $41-50$ year age group. The mean \pm standard deviation were $36.9 \pm 8.7$ year. Among non-smokers majority (43.3\%) were aged between 21 - $30 \mathrm{yr}$, while among smokers majority (51.1\%) were aged between 
41 - 50 yr. As the age goes on increasing so does percentage of smokers. Most (48.9\%) of the smokers fell into the moderate smoker (11-15 cigarette per (figure 2).

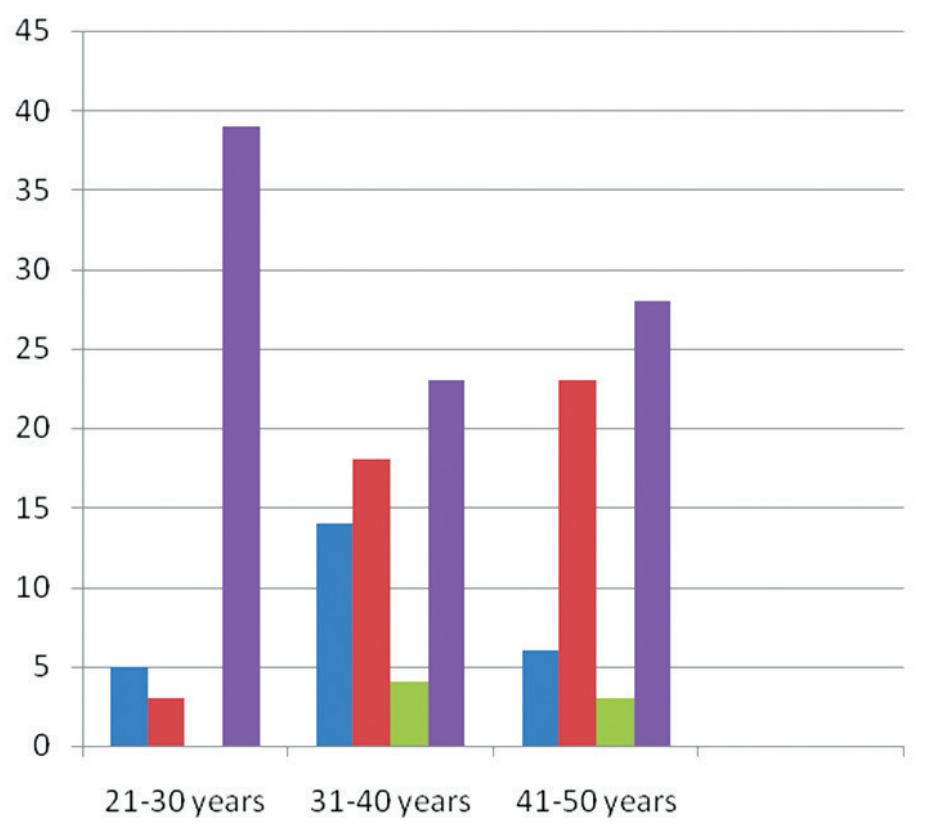

- Mild (1-10 cigrettes/day)

noderate (11-15 cigrettes/day)

a Severe(16-20 cigrettes/day)

non smokers

Figure 1: Comparison of age and severity of smoking among smokers

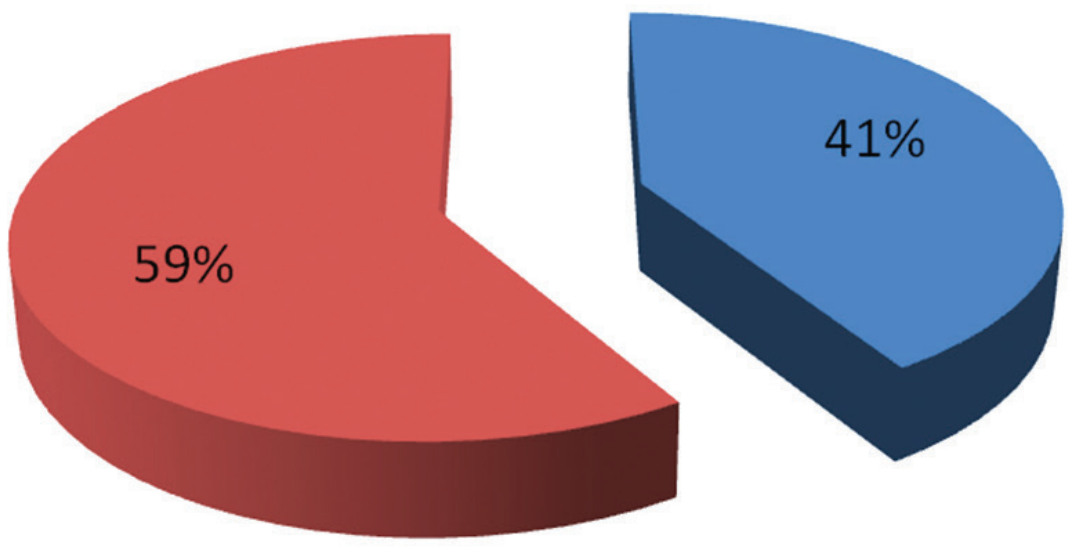

- $<=10$ years

- $>10$ years

Figure 2: Duration of smoking among smokers 
Table 1: Comparison of LIPID profile in non- smokers and smokers

\begin{tabular}{lllllc}
\hline \multicolumn{1}{c}{ LFT } & Smoking habit & N & Mean & SD & P-value \\
\hline \multirow{2}{*}{ Cholesterol } & Non-smoker & 90 & 4.174 & 0.840 & \multirow{2}{*}{0.001} \\
& Smoker & 90 & 4.632 & 1.037 & \\
\multirow{2}{*}{ Triglycerides } & Non-smoker & 90 & 1.443 & 0.883 & 0.090 \\
& Smoker & 90 & 1.689 & 1.046 & \\
LDL & Non-smoker & 90 & 2.515 & 0.809 & \multirow{2}{*}{0.007} \\
& Smoker & 90 & 2.848 & 0.833 & \\
VLDL & Non-smoker & 87 & 0.685 & 0.654 & \multirow{2}{*}{0.351} \\
& Smoker & 90 & 0.764 & 0.464 & \\
HDL & Non-smoker & 90 & 1.077 & 0.562 & 0.179 \\
\hline
\end{tabular}

To compare the LIPID profile of non-smokers and 0.833 . The mean LDL level was found statistically smokers, unpaired student's t-test was applied. It significantly more among smokers. The mean \pm SD was observed that the mean \pm SD of the cholesterol of the VLDL level in the smoker was $0.764 \pm 0.464$. level among smokers was $4.632 \pm 1.037$. The mean The mean VLDL level was found more in the smoker. cholesterol level found statistically significantly more But the difference is not statistically significant. among smokers. The mean \pm SD of the Triglycerides The mean \pm SD of the HDL level in the smoker was level in the smoker was $1.689 \pm 1.046$. The mean $0.990 \pm 0.235$. The mean HDL level was found less Triglycerides level found more among smokers. But in the Smoker. But the difference is not statistically the difference is not statistically significant. The mean significant (table 1).

$\pm \mathrm{SD}$ of the LDL level in the smoker was $2.848 \pm$

Table 2: Comparison of lipid profile in smokers according to no. of cigarette smoked/day

\begin{tabular}{llllcc}
\hline \hline Lipid constituent & \multicolumn{1}{c}{ Severity of smoking } & N & Mean & Std. Dev. & P-value \\
\hline \multirow{3}{*}{ Cholesterol } & $1-10$ (Mild smoker) & 25 & 4.676 & 1.275 & \\
& $11-15$ (Moderate smoker) & 44 & 4.586 & 0.882 & 0.921 \\
& $16-20$ (Severe smoker) & 21 & 4.676 & 1.072 & \\
Triglycerides & $1-10$ (Mild smoker) & 25 & 1.432 & 1.221 & \\
& $11-15$ (Moderate smoker) & 44 & 1.573 & 0.877 & 0.018 \\
& $16-20$ (Severe smoker) & 21 & 2.238 & 0.998 & \\
LDL & $1-10$ (Mild smoker) & 25 & 3.004 & 0.943 & \\
& $11-15$ (Moderate smoker) & 44 & 2.868 & 0.681 & 0.291 \\
& $16-20$ (Severe smoker) & 21 & 2.619 & 0.968 & \\
VLDL & $1-10$ (Mild smoker) & 25 & 0.660 & 0.536 & \\
& $11-15$ (Moderate smoker) & 44 & 0.705 & 0.385 & 0.017 \\
& $16-20$ (Severe smoker) & 21 & 1.012 & 0.459 & \\
HDL & $1-10$ (Mild smoker) & 25 & 0.976 & 0.240 & \\
& $11-15$ (Moderate smoker) & 44 & 1.014 & 0.206 & 0.628 \\
& $16-20$ (Severe smoker) & 21 & 0.957 & 0.286 & \\
\hline
\end{tabular}


The mean cholesterol level found statistically not significantly different among the pre-defined three types of smokers. The mean Triglycerides level found statistically significantly different among the three types of smokers. It varies with a low of 1.432 in the mild smoker to a high of 2.238 in the severe smoker. The mean LDL level found statistically not significantly different among the three types of smokers. The mean VLDL level found statistically significantly different) among the three types of smokers. It varies with a low of 0.660 in the mild smoker to a high of 1.012 in the severe smoker. The mean HDL level found statistically not significantly different among the three types of smokers (table 2).

\section{Table 3: Comparison of LIPID profile in smokers according to duration of smoking}

\begin{tabular}{lccccc}
\hline \multicolumn{1}{c}{ Lipid constituent } & Duration of smoking & N & Mean & Std. Dev. & P-value \\
\hline \multirow{2}{*}{ Cholesterol } & $<=10 \mathrm{yr}$ & 37 & 4.538 & 1.073 & \multirow{2}{*}{0.474} \\
\multirow{2}{*}{ Triglycerides } & $>10 \mathrm{yr}$ & 53 & 4.698 & 1.015 & \\
& $<=10 \mathrm{yr}$ & 37 & 1.581 & 1.179 & \multirow{2}{*}{0.417} \\
LDL & $>10 \mathrm{yr}$ & 53 & 1.764 & 0.946 & \\
& $<=10 \mathrm{yr}$ & 37 & 2.835 & 0.786 & \multirow{2}{*}{0.905} \\
VLDL & $>10 \mathrm{yr}$ & 53 & 2.857 & 0.871 & \\
& $<=10 \mathrm{yr}$ & 37 & 0.704 & 0.518 & \multirow{2}{*}{0.312} \\
HDL & $>10 \mathrm{yr}$ & 53 & 0.806 & 0.423 & \\
& $<=10 \mathrm{yr}$ & 37 & 0.995 & 0.231 & \multirow{2}{*}{0.878} \\
\hline
\end{tabular}

It was observed that in each of the five parameters viz., Cholesterol, Triglycerides, LDL, VLDL, and HDL there is no statistically significantly difference in mean level. While in all first four parameters viz., Cholesterol, Triglycerides, LDL and VLDL the mean levels are a bit higher in the more than 10 year group as compared to at most 10 year group, the mean HDL level was minimum for more than 10 year as compared to at most 10 year group (table 3).

\section{DISCUSSION}

In our study the 2 group of participants i.e. smokers and non smokers were of comparable age. All participants were non diabetic and normotensive. Dyslipidemia is a well-established risk factor for the development of coronary artery disease. Our study concluded that the lipid profile is drastically altered in case of smokers compared to non smokers leading to increased propensity to coronary artery disease.

Smokers in our study have a lower HDL compared to non smokers which can be attributed to the fact that smoking causes fall in estrogen levels which leads to decreased HDL levels. Similar findings were observed in a study conducted by Joshi et al. ${ }^{7}$ and Zamirand et al. ${ }^{8}$
Similarly in our study we found that triglycerides, IDL, LDL levels are significantly raised in smokers which is similar to findings of the study conducted by many authors ${ }^{7,9-13}$

The mean LDL cholesterol in mild/moderate and severe smokers was significantly higher i.e. a positive correlation between smokers and the number of cigarette/ beedi smoked per day. The nicotine contained in cigarette increases the circulatory pool of atherogenic LDL through accelerated transfer of lipids from HDL and impaired clearance of LDL from plasma compartment and hence LDL cholesterol in the arterial wall is increased. These findings are similar to the study conducted by Neki et al. ${ }^{14}$ and Zamirand et al. ${ }^{8}$

The values of serum triglycerides, cholesterol and VLDL were higher in those subjects smoking cigaretts/bidis for more than 3 pack years. However th values are not statistically significant. Nicotine causes increased activity of the adrenal sympathetic system leading to increased release catecholamines which causes increased lipolysis and thereby increased concentration of plasma free fatty acids which causes further increased secretion of hepatic triglyceride secretion along with VLDL in blood stream. Presence of hyperinsulinemia also leads to increased cholesterol, LDL and cholesterol due to 
increased activity of lipoprotein lipase. These changes contribute to increased atherosclerotic damage. These findings are similar to the study done by Rosesnson ${ }^{15}$ and Neeki et al. ${ }^{14}$

The values of serum triglycerides, cholesterol and VLDL were higher in those subjects smoking cigaretts/bidis for more than 15 years as compare to those of 5-10 years or 1-5 years but the difference was not statistically significant. However a larger sample could be more predictive of the more accurate inference.

Since our study was of cross sectional nature the association with risk factors like smoking could not studied extensively. A prospective cohort study would be best.

\section{CONCLUSIONS}

Smoking is a major risk factor for development of cardiovascular diseases. Smoking causes significant alterations in the lipid profile. Total serum Cholesterol, LDL, VLDL, triglycerides were significantly higher in smokers as compared to non smokers however serum HDL was significantly lower in smokers. Alterations in lipid profile were found to be directly correlated with the number of cigarettes smoked daily whereas the duration of smoking had no significant correlation with alterations in lipid profile.

\section{REFERENCES}

1. Mattila KJ, Valle MS, Nieminen MS, Valtonen VV, Hietaniemi KL. Dental infections and coronary atherosclerosis. Atherosclerosis. 1993;103(2):205-11.

2. Austin MA. Plasma tryglicerides and coronary heart disease. ArterioThrom 1991;11;2-14.

3. Carlson LA, Bottiger LE, Ahfeldt PE. Risk factors for myocardial infarction in the Stockholm prospective study: A 14 year follow-up on focusing on the roleof plasma triglycerides and cholesterol. Acta Med scand. 1979; 206; 315-60.

4. Brischetto CS, Connor WE. Plasma lipid and lipoprotein profiles of cigarette smokers. American journal of cardiology. 1983;52(7):675-80.

5. Cooper T. Cardiovascular diseases, the health consequences of smoking; Atlanta ; US Dept new Public Health Service Center for Diseases control. 1975:13-38.
6. Pairat Saengdith. Effects of cigarette smoking on serum lipids among priests in Bangkok. Journal of medicine assoc Thai. 2008;91:41-4.

7. Joshi N, Shah C, Mehta HB, Gokhle PA. Comparative study of lipid profile on healthy smokers and non smokers. Int Med sci Public Health 2013; 2:662-626.

8. Zamir MAA, Muahmmed SB, Muhammed S. Lipid Profile in Smoking. JAMC. 2000;12(3):19-21.

9. Afshan A, Sugoor M, Swati IA, Patil RB. A hospital based study on lipid profile in smokers and non-smokers-a comparative study. Journal of Evolution of Medical and Dental Sciences. 2012;1(5):662-7.

10. Yadav BK, Bade AR, Singh J, Jha B. Comparative study of lipid profile in smokers, tobacco chewers and diabetic patients. Journal of Institute of Medicine. 2007;27(3).

11. Fariduddin JM, SS M. A comparative study on lipid profile and uric acid among smokers and non-smokers. International Journal of Clinical Biochemistry and Research. 2016;3(4):469-71.

12. Reddy AV, Killampalli LK, Prakash AR, Naag S, Sreenath G. Analysis of lipid profile in cancer patients, smokers, and nonsmokers. Dental research journal. 2016;13(6):494

13. Sonagra AD, Shylaja TV, Makandar A, ZD. Study of Lipid Profile among Healthy Smokers and Non Smokers. International Journal of Biotechnology and Biochemistry. 2017;3(1):87-94.

14. Neki NS. Serum Lipids in Patients with Oral Tobacco Habits and Oral Precancerous Lesions and Conditions. Journal. Indian Academy of Clinical Medicine. 2002;3:51-4.

15. Rosenson RS. Low level of HDL cholesterol (Hypoalphalipoproteinemia), an approach to management. Arch Intern Med. 1993;153(13):1528-40.

\section{How to cite this article?}

Byar NK, Shrestha TM, Pratap NP, Khanal S. A Comparative Study of Lipid Profile among Smokers and Non Smokers. Journal of Karnali Academy of Health Sciences. 2019;2(1):4-9

Conflict of Interest: None Source of Support: None 